\title{
Auto-Titration Technique Using Intracranial Self-Stimulation and Effects of Antianxiety Drugs
}

\author{
Yasuyuki ICHIMARU, Minehiro MORIYAMA and Yutaka GOMITA* \\ Department of Pharmacology. Daichi College of Pharmaceutical Sciences. \\ 22-1 Tamagawa-cho. Minami-ku. Fukuoka 815, Japan \\ Accepted July 29. 1985
}

\begin{abstract}
In order to study effects of antianxiety drugs on the threshold of intracranial self-stimulation (ICSS), the auto-titration technique was used in the two lever Skinner box. This procedure consisted of conventional ICSS except the brain stimulation current intensity was decreased after every 20 lever press for ICSS, and the animal could press the another reset lever (at any time) to reset the stimulation current intensity to the preseted level. The current intensity at which the animal pressed the reset lever (reset current) was defined as the threshold of ICSS. Since reset currents that the animals showed were very stable during this experiment, the effects of chlordiazepoxide, diazepam and meprobamate were studied. The reset current was significantly lowered by $5.0 \mathrm{mg} / \mathrm{kg}$ chlordiazepoxide, p.o., at $1 \mathrm{hr}$ after administration accompanied by the significant increase of ICSS. and the reset current was significantly lowered by $20.0 \mathrm{mg} / \mathrm{kg}$ of chlordiazepoxide. On the other hand, diazepam did not lower the reset current, although a significant increase of ICSS was observed at $1.0 \mathrm{mg} / \mathrm{kg}$. p.o. The reset current was lowered by meprobamate at $100 \mathrm{mg} / \mathrm{kg}$. P.O. accompanied by the increase of ICSS, and a significant increase of ICSS was observed at $200 \mathrm{mg} / \mathrm{kg}$ but not accompanied by lowered reset current. From these results, increased susceptibility to the brain stimulation current may be involved in the facilitating effects of chlordiazepoxide and meprobamate on ICSS.
\end{abstract}

In experimental animals, antianxiety drugs such as benzodiazepines exert various effects such as sedation, potentiation of hypnotics, muscle relaxation, and anti-convulsive and anti-conflict actions (1-3). Moreover, there are several studies showing that response rates of intracranial self-stimulation (ICSS) were facilitated by antianxiety drugs (4-7). Some of these studies suggest that antianxiety drugs may enhance the susceptibility to brain stimulation reward, although no direct evidence has been provided. That is to say, a critical question arises as to whether such facilitation is due to a change of the rewarding effect of brain stimulation itself and/or due to an alteration in motor activity.

\footnotetext{
* Present address: Department of Hospital Pharmacy, Okayama University Medical School, 2-5-1. Shikata-cho, Okayama 700, Japan.
}

arousal level and so on.

Recently, Schacfer and Michael (8) studied the effect of various neuroleptics on ICSS threshold by using the auto-titrating brain self-stimulation technique for avoiding the confounding effect on ICSS. This autotitration (self-determination) technique may be available to clarify the facilitating effects of antianxiety drugs. In several studies using this technique, the effects of drugs such as catecholamine releasers, neuroleptics, GABA agonists and opiate on the threshold of ICSS were reported $(9-11)$. However, the effects of antianxiety drugs have not been examined yet. In the present study, therefore, the possibility that antianxiety drugs lowered the threshold of brain stimulation reward was studied by means of the auto-titration technique. 


\section{Materials and Methods}

Animals: Male Wistar strain rats weighing 250-300 g at the surgery were used in this experiment. Two or three animals were housed in each conventional plastic walled cage, at an ambient temperature of $21-25^{\circ} \mathrm{C}$ and a relative humidity of $60 \%$, and they were given food and water ad libitum. The animal room was illuminated between 0900 and 2100 .

Surgery and histology: All animals were anesthetized with sodium pentobarbital 50 $\mathrm{mg} / \mathrm{kg}$, i.p., and chronically implanted with a bipolar stainless steel electrode $(250 \mathrm{ftm}$ in diameter, insulated except at a tip). The electrode was aimed at the lateral hypothalamic region $(A, 5.4 ; \mathrm{L}, 1.8 ; \mathrm{H},-3.0 \mathrm{~mm}$ ) using de Groot's coordinates (12). All animals were intramuscularly given penicillin at 150.000 units/animal after the surgery. At least 10 days were allowed for recovery before training for ICSS.

At the end of the experiment, all animals were given an overdose of sodium pentobarbital. The head was intracardially perfused with physiological saline and then $10 \%$ formalin. The brain was immersed in formalin-saline solution for at least 10 days. Each brain was frozen, and $40 \mu \mathrm{m}$ slices were made and mounted on glass slides following by staining with cresyl-violet. The localization of implanted electrode tips in the lateral hypothalamus was verified by the inspection of stained sections.

Apparatus: The experiment was carried out in a conventional Skinner box $(30.4 \mathrm{~cm}$ wide. $25.4 \mathrm{~cm}$ long and $27.7 \mathrm{~cm}$ high) with two levers. One lever for brain-stimulation (BSL) was positioned $4.5 \mathrm{~cm}$ above the grid floor at the right side of the side wall of the box and protruded $2.5 \mathrm{~cm}$ into the box, and another lever for reset (RL) was positioned at the left side of same wall at $20 \mathrm{~cm}$ apart from the former lever.

Procedure: The auto-titration technique used in this experiment was similar to that reported by Schaefer et al. (13), but with several modifications. A press of the BSL activated a counter and resulted in a brain stimulation through an electric stimulator (WES-101). The stimulator delivered the negative square wave pulses (at frequency $100 \mathrm{pulses} / \mathrm{sec}$, duration of $0.2 \mathrm{sec}$ ) corresponding with each time the BSL was pressed. The stimulator had two functionally different modes, i.e., an ICSS mode and an auto-titration mode. In the ICSS mode, a fixed intensity of brain stimulation current was delivered by each press of the BSL. On the other hand, in the auto-titration mode, the intensity of the delivered stimulation current was dropped stepwise 2. 5 or $100 \%$ of the previous intensity after a preset number (1-90) of BSL presses was made; and by pressing the $R L$, the decreased intensity of current was returned to the initial intensity level accompanied with a contingent cue that the pilot lamp was on. The pressing of the $R L$ never caused brain stimulation. The record of the press of $B S L$ was performed by a cumulative recorder (WES-101-R) and a digital counter, and in the auto-titration mode, a recording pen was returned to the zero position by pressing the RL.

Following recovery from the implantation surgery. each animal was trained to press the BSL for ICSS of the lateral hypothalamus according to the usual procedure. After the responses for brain stimulation under a continuous reinforcement schedule reached a specific criterion (more than 1000 responses/ 15 min) through three successive days, the animal was trained to press the RL. In this training, the intensity of brain stimulation current was dropped $100 \%$ to zero at each 50 th press of the BSL and returned to the initial intensity when the animal pressed the RL. After the stable reset response was obtained in reset training. the animal was exposed to the auto-titration procedure. In this procedure, after every 20th press of the BSL, the stimulation current was decreased by $2 \%$. That is to say, the animal pressed the BSL while the stimulation current was effective to reinforce the behavior, and pressed the $R L$ when the stimulation current might be no longer ineffective. Then. the stimulation current when the animal pressed the RL was referred to the "reset current". A number of daily training sessions (15 min) were given to each animal.

In each test, we recorded the animal's response for $15 \mathrm{~min}$ before drug adminis- 
tration and then again for a $15 \mathrm{~min}$ period at 1,2, 4 and $24 \mathrm{hr}$ after administration. Five to 7 rats were used in each dose of drugs or vehicle. At least 2 weeks elapsed between each drug administration when administered to the same animal.

Drugs: The drugs used were chlordiazepoxide hydrochloride (Hoechst) which was dissolved in physiological saline and diazepam (Kodama) and meprobamate (Daiichi) which were each dissolved in $0.5 \%$ carboxymethylceliulose- $\mathrm{Na}$ solution and administered orally.

Statistical analysis: The statistical analysis of the experimental results was made by the Mann-Whitney $U$-test (14).

\section{Results}

All animals that showed ICSS were able to auto-titrate their own stimulation current. The three representative daily reset currents are shown in Fig. 1. As shown in the figure. the daily reset current of each case was stable through training sessions with little variation.

A representative cumulative record of the rat (No. 120) treated with chlordiazepoxide $20.0 \mathrm{mg} / \mathrm{kg}$, p.o., is shown in Fig. 2. The reset current was lowered (i.e., each peak point of the cumulative record was raised) at 1 and
$2 \mathrm{hr}$ after the administration of chlordiazepoxide and recovered at $24 \mathrm{hr}$ after. The effects of $5.0-20.0 \mathrm{mg} / \mathrm{kg}$ chlordiazepoxide. p.o., on the reset current and the numbers of BSL presses are shown in Fig. 3. The reset current was significantly decreased at $1 \mathrm{hr}$ after administration at doses of 5.0 and 20.0 $\mathrm{mg} / \mathrm{kg}(\mathrm{U}=6$ and $11, \mathrm{P}<0.05$, respectively). and it was slightly decreased at $10.0 \mathrm{mg} / \mathrm{kg}$. but not significantly (upper pannel of Fig. 3). On the other hand, as shown in the lower pannel of Fig. 3, the numbers of BSL presses

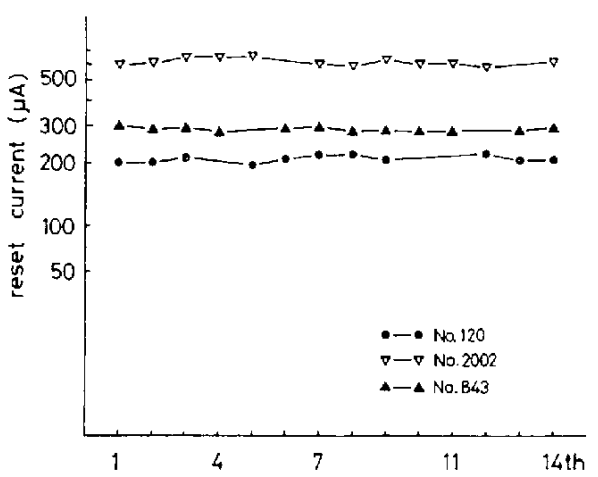

Fig. 1. Daily changes of reset current. Reset currents of three animals (No. 120,843 and 2002) are shown. Vertical: mean of reset current, Horizontal: days.
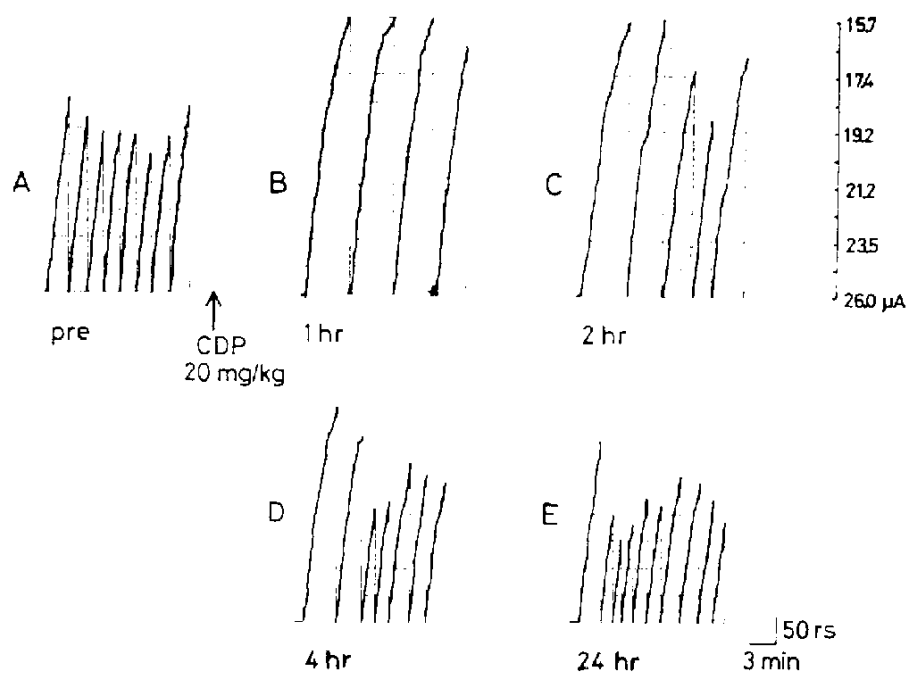

Fig. 2. Representative cumulative records of chlordiazepoxide on auto-titration behavior. A: before administration. B, C, D and E: 1,2, 4 and $24 \mathrm{hr}$ after administration, respectively. Stimulation current (rat No. 120) are indicated on the left side. 

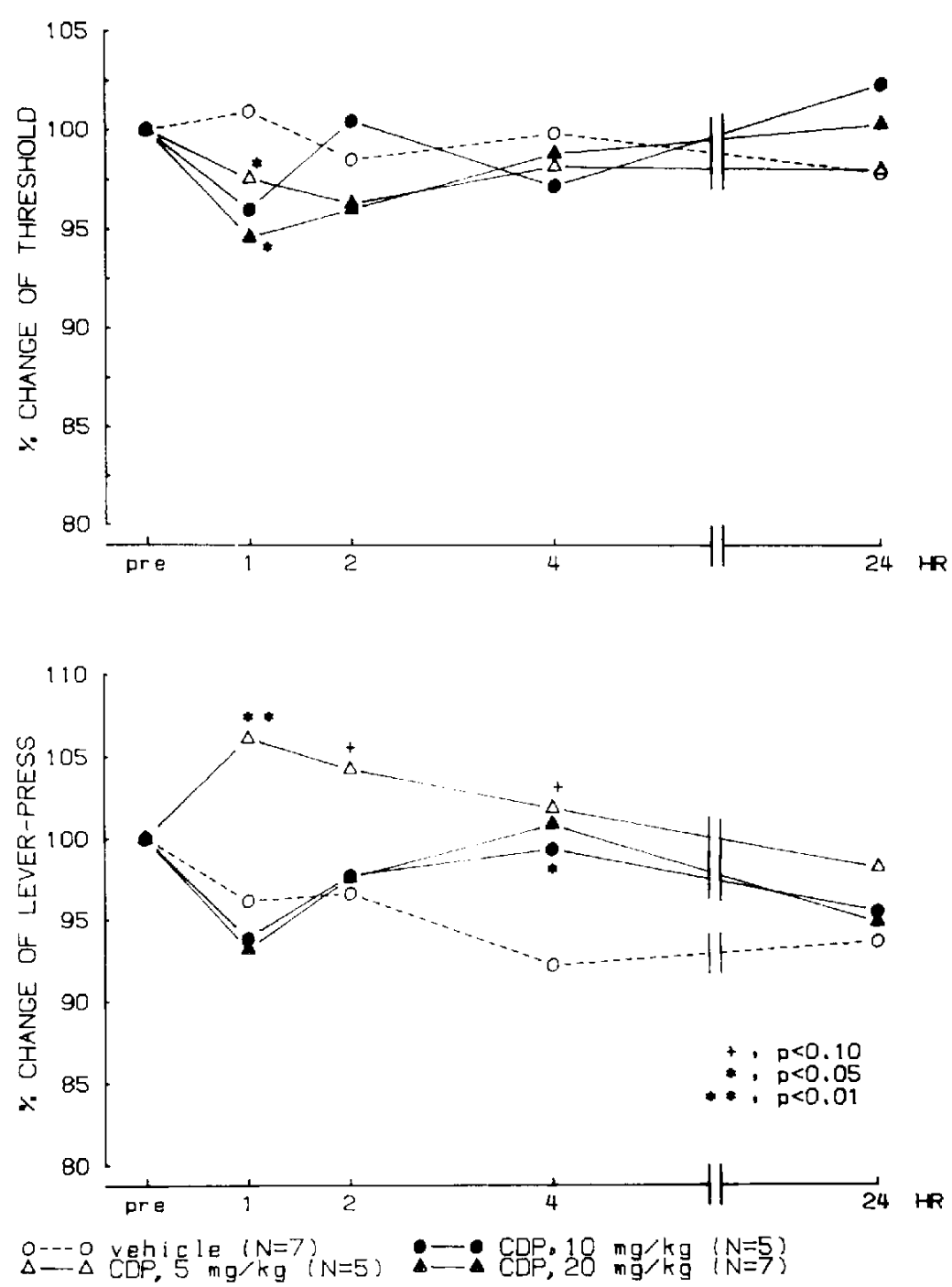

Fig. 3. Effect of chlordiazepoxide on auto-titration behavior. Time course of reset current (upper pannel) and number of lever pressing responses (lower pannel) are shown as \% change from the mean of three successive days before drug administration. CDP: chlordiazepoxide

for 15 min were significantly increased $(U=3$. $\mathrm{P}<0.05$ ) at $1 \mathrm{hr}$ after the administration of $5.0 \mathrm{mg} / \mathrm{kg}$ and showed tendencies to increase at 2 and 4 hr after $(U=9$ and $8, P<0.10$. respectively). These were significantly increased $(U=5, P<0.05)$ at $4 \mathrm{hr}$ after adminis tration of $10.0 \mathrm{mg} / \mathrm{kg}$. However, at a dose of $20.0 \mathrm{mg} / \mathrm{kg}$, no significant change was obtained, and the animals showed muscle relaxations.

The effects of diazepam $1.0-10.0 \mathrm{mg} / \mathrm{kg}$. p.o. On reset current and the numbers of the press of BSL are shown in Fig. 4. The reset current was not affected by the treatments with diazepam at any dose. On the other hand, numbers of BSL presses were significantly increased at $1 \mathrm{hr}$ and $2 \mathrm{hr}(\mathrm{U}=2$, $P<0.01, U=6, P<0.05$, respectively) after administration of $1.0 \mathrm{mg} / \mathrm{kg}$ of diazepam. Tendencies to increase were obtained at $4 \mathrm{hr}$ after administration of $2.0 \mathrm{mg} / \mathrm{kg} \quad(U=8$. $\mathrm{P}<0.10$ ) and $1 \mathrm{hr}$ after administration of 

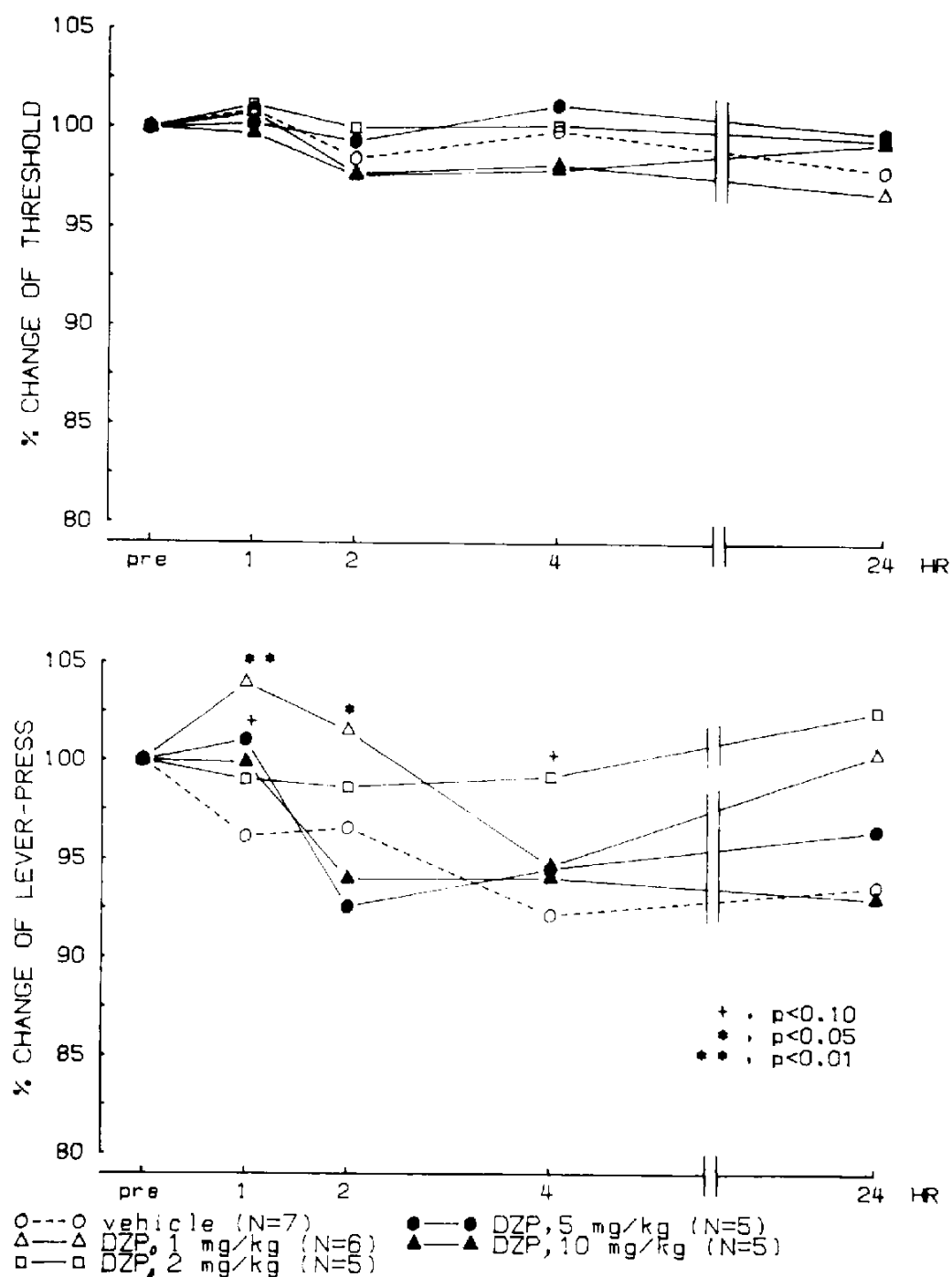

Fig. 4. Effect of diazepam on auto-titration behavior. Time course of reset current (upper pannel) and number of lever pressing responses (lower pannel) are shown as \% change from the mean of three successive days before drug administration. DZP: diazepam

$5.0 \mathrm{mg} / \mathrm{kg}(U=7, P<0.10)$. At the highest dose of $10.0 \mathrm{mg} / \mathrm{kg}$ of diazepam, the animals showed muscle relaxation.

The effects of 100 and $200 \mathrm{mg} / \mathrm{kg} \mathrm{me}$ probamate, p.o., on reset current and numbers of BSL presses are shown in Fig. 5. The reset current was slightly decreased at $2 \mathrm{hr}$ after the administration of $100 \mathrm{mg} / \mathrm{kg}$, but not significantly. The numbers of BSL presses were significantly increased at $1 \mathrm{hr}$ after administration of 100 and $200 \mathrm{mg} / \mathrm{kg}(\mathrm{U}=7$ and $8, P<0.05$, respectively).

\section{Discussion}

Previously, we and others (4-7) have reported that antianxicty drugs facilitated the response rate of ICSS; however, the mechanism of these facilitating effects by antianxiety drugs is not clarified yet. Some of the possible mechanisms are 1) enhancements of susceptibility to brain stimulation reward as suggested by Stark et al. (15), 2) 

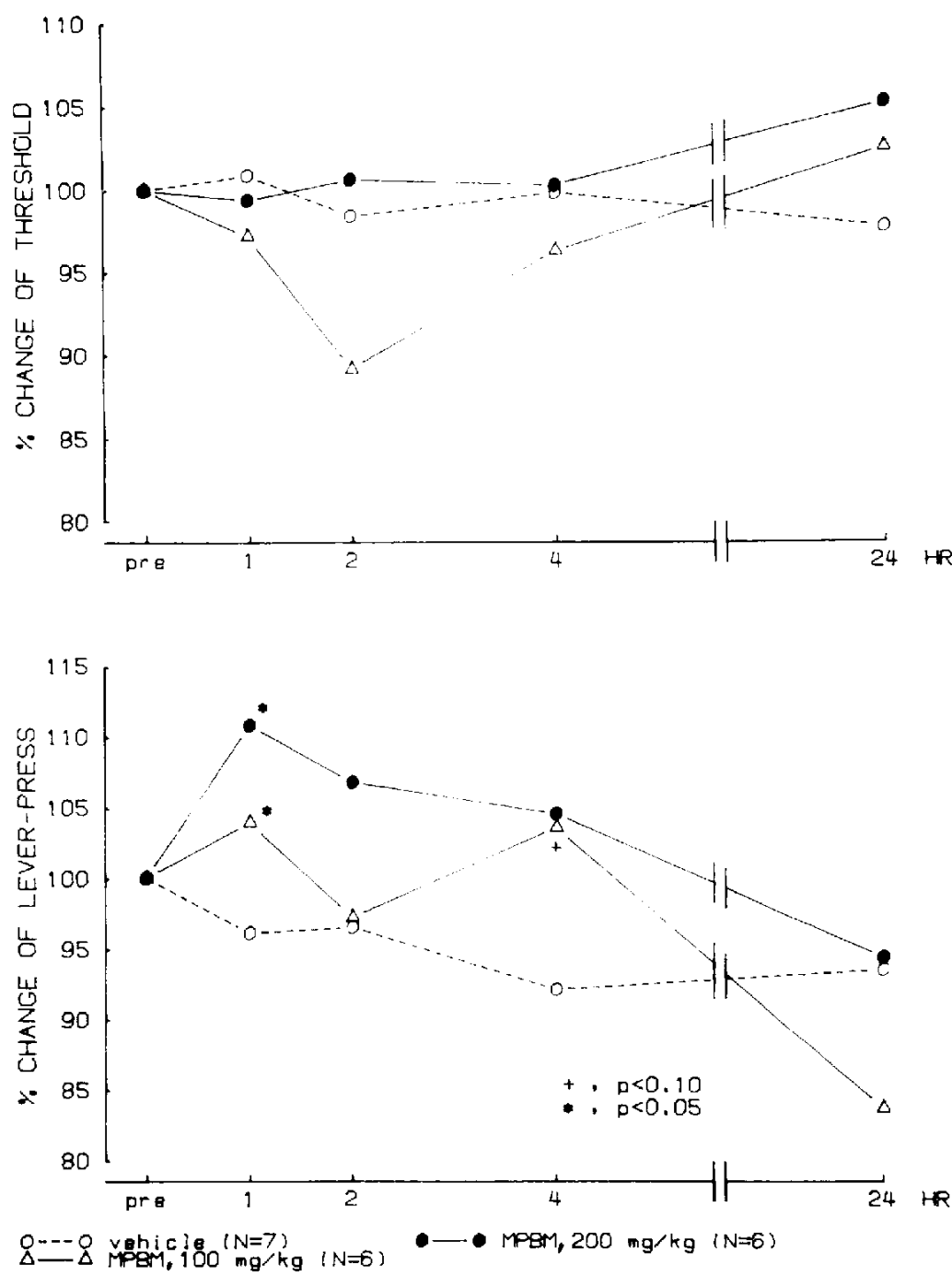

Fig. 5. Effect of meprobamate on auto-titration behavior. Time course of reset current (upper pannel) and number of lever pressing responses (lower pannel) are shown as $\%$ change from the mean of three successive days before drug administration. MPBM: meprobamate

inhibitions of aversive components that accompanied brain stimulation revard as suggested by Olds (7), and 3 ) non-specific actions to general behaviors of animals such as enhancements of locomotor activities as suggested by Olds (7). In the present experiment, we investigated the first possibility by means of auto-titration (selfdetermination) of brain stimulation current. In this auto-titration technique, it is hypothetized that the reset current means the threshold of brain stimulation reward.

In the present study, the reset currents that animals titrated were stable through the experimental periods. Therefore, this procedure is useful for determining effects of drugs on suscepribility to brain stimulation reward. Moreover, the response rate in this technique consisted of various components of response rate, i.e. high rate response at high stimulation current to low response rate at low current during reset and reset intervals. 
We reported previously that the low rate responses at low current stimulation were available to investigate the facilitating effect of drugs on the ICSS (4). Therefore, this auto-titration procedure may also be useful for estimating the facilitating effect of drugs on the ICSS. Howcver, there are some differences between responses in the present procedure and low rate responses at low current stimulation. since responses in autotitration consisted of various components of response rates. For example, the facilitatıng effect of chlordiazepoxide was observed at $10.0 \mathrm{mg} / \mathrm{kg}$, p.o., in low rate responses at low current stimulation, and the inhibitory effect was observed at $20.0 \mathrm{mg} / \mathrm{kg}$, p.o. (4). In auto-titration procedure, on the other hand, the facilitating effect of chlordiazepoxide was observed only at $5.0 \mathrm{mg} / \mathrm{kg}$, p.o., and no significant effect was observed at 10.0 and $20.0 \mathrm{mg} / \mathrm{kg}$. p.o. Furthermore, we already reported that chlordiazepoxide facilitated the lateral hypothalamic ICSS through 5.0 to $20.0 \mathrm{mg} / \mathrm{kg}$. p.o., in the short delayed (1-1.5 sec) DRL schedule (16). From these observations, the facilitating effect of minor tranquilizers may be depend on the schedule employed.

When effects of antianxiety drugs were investigated in this technique. chlordiaze poxide and meprobamate showed enhancements of susceptibility (the lowered threshold) to brain stimulation reward. although diazepam had little effect. Although the facilitating effects of three antianxiety drugs on ICSS responses were also confirmed in the present experiment, the threshold lowering effect of diazepam was not observed. We do not have a proper explanation for these results, but it can be thought that the threshold lowering effect of diazepam was not detected by the present procedure, because the facilitating effect of diazepam was weaker than that of chlordiazepoxide in the present experiment and another report (17)

These results indicate that the facilitating effects of antianxiety drugs when administered on an acute basis might be explained by the first possibility mentioned above (i.e., the enhancement of susceptibility to brain stimulation). Concerning effects of chlor- diazepoxide and meprobamate, at least, the first possibility may be involved in the mechanisms of the facilitating effect on ICSS. Therefore, the latter two possibilities must be investigated later, especially concerning the effects of diazepam. In addition, as the present data are based on the ICSS of hypothalamic region, it is also unknown whether these possibilities are applicable to the other ICSS regions.

Acknowledgement: This research was supported In part by a Grant-in-Aid (No. 58771688 to Yl) for Scientific Research from the Ministry of Education. Science and Culture of Japan.

\section{References}

1 Kales, A.: Benzodiazepines in the treatment of insomnia. In Pharmacology of Benzodiazepines. Edited by Usdin, E., Skolnick, P., Tallman, J.F., Jr., Greenblatt, D. and Paul, S.M., p. 199-217. MacMillan, London (1982)

2 Haefley, W., Kulcsar, A., Möhler, H., Pieri, L., Polc, P. and Schaffner, R.: Possible involvement of GABA in the central action of benzodiazepines. Adv. Biochem. Psychopharmacol. 14, 131-151 (1975)

3 Geller, I. and Seifter, J.: The effects of meprobamate, barbiturates, d-amphetamine and promazine on experimentally induced conflict in the rat. Psychopharmacology (Berlin) 1, 482492 (1960)

4 Gomita, Y., Ichimaru, Y. and Moriyama, M.: Effects of benzodiazepines on low rate responding for low current brain stimulation reward. Japan. J. Pharmacol. 33, 498-502 (1983)

5 Ichimaru, Y., Moriyama, M. and Gomita, Y.: Effects of antianxiely and antipsychotic drugs on DRL responding for brain stimulation. Life Sci. $32,437-448$ (1983)

6 Domino, E.F. and Olds, M.E.: Effects of $d$ amphetamine, scopolamine. chlordiazepoxide and diphenylhydantoin on self-stimulation behavior and brain acetylcholine. Psychopharmacology (Berlin) 23, 1-16 (1972)

7 Olds, M.E.: Facilitatory action of diazepam and chlordiazepoxide on hypothalamic reward behavior. J. Comp. Physiol. Psychol. 62, $136-$ 140 (1966)

8 Schaefer, G.J. and Michael, R.P.: Acute effects of neuroleptics on brain self-stimulation threshold in rat. Psychopharmacology (Berlin) 67. 9-15(1980)

9 Stein. L. and Ray, O.S.: Brain stimulation reward 
"thresholds" self-determined rat. Psychopharmacology (Berlin) 1, 251-256 (1960)

10 Nazzaro, J. and Gardner, E.L.: GABA antagonısm lowers self-stimulation thresholds in the ventral tegmental area. Brain Res. 189, 279-283 (1980)

11 Kornetsky, C., Esposito, R.U., MacLean, S. and Jacobson, J.O.: Intracranial self-stimulation threshold. Arch. Gen. Psychiat. 36, 289-29? (1979)

12 de Groot, J.: The rat brain in stereotaxic coordinates. Verh. K. Ned. Akad. Wet. 52, 14-24 (1959)

13 Schaefer, G.J., Baumgardner, D.G. and Michael, R.P.: Constant-current, biphasic titrating stimulator for brain self-stimulation. Physiol. Behav. 22, 1217-1219 (1979)

14 Siegel, S.: Non-parametric statistics for the behavioral sciences. McGraw Hill. New York (1956)

15 Stark, P., Turk, J.A., Redman, C.E, and Henderson, J.K.: Sensitivity and specificity of positive reinforcing areas to neurosedatives, antidepressants and stimulants. J. Pharmacol. Exp. Ther, 166, 163-167 (1969)

16 Ichimaru, Y., Moriyama, M. and Gomita, Y.: Effects of psychotropic drugs on DRL-response to brain stimulation reward in rats (II). Japan. J. Pharmacol. 32, Supp. 34P (1982)

17 Gomita, Y., Morii, M., Ichimaru, Y., Moriyama, M. and Ueki, S.: Studies on behavioral and electroencephalographic effects of clobazam. Folia Pharmacol. Japon. 82, 267-292 (1983) (Abs. in English) 